\title{
Icariin Alleviates Glucocorticoid-Induced Osteoporosis through EphB4/Ephrin-B2 Axis
}

\author{
Mi Huang $\left(\mathbb{D},{ }^{1}\right.$ Ying Wang, ${ }^{2,3}$ and Rui Peng $\mathbb{D}^{1}$ \\ ${ }^{1}$ Hubei University of Chinese Medicine, Hubei, Wuhan 430000, China \\ ${ }^{2}$ South China Botanical Garden, Chinese Academy of Sciences, \\ Key Laboratory of South China Agricultural Plant Molecular Analysis and Genetic Improvement, \\ Key Laboratory of Guangdong Province Applied Botany, Guangzhou 510650, China \\ ${ }^{3}$ Gannan Normal University, Ganzhou, Jinagxi 341000, China
}

Correspondence should be addressed to Rui Peng; 974831374@qq.com

Received 21 February 2020; Accepted 1 May 2020; Published 18 May 2020

Guest Editor: Reggiani Vilela Gonçalves

Copyright (c) $2020 \mathrm{Mi}$ Huang et al. This is an open access article distributed under the Creative Commons Attribution License, which permits unrestricted use, distribution, and reproduction in any medium, provided the original work is properly cited.

\begin{abstract}
Purpose. Glucocorticoid (GC) is the most important risk factor for osteoporosis (OP); in the present study, we examined the potential mechanism of icariin, a natural bioactive compound isolated from the traditional Chinese herbal Epimedium, for GCinduced OP to explore its potential therapeutic effect. Methods. We used a GC-induced OP mice model and treated with icariin. Pathological changes were measured by H\&E staining, and the effects of icariin on osteoblasts and osteoclasts were measured by immunohistochemistry (IHC) staining and western blot (WB) analyses, while trabecular bone parameters were detected by microCT imaging in vivo. Results. The results showed that in GC-induced OP symptoms, icariin treatment significantly increased the density of the trabecular bone when exposed to GC, revealed by H\&E staining and micro-CT imaging. IHC staining showed that GC-induced OP had a lower EphB4 expression and higher Ephrin-B2 expression, but icariin could promote EphB4 while suppressing Ephrin-B2 expression. The WB results also provided evidence of the same protein expression trend, showing that the osteoblast marker OCN and the EphB4 downstream factor RhoA in the GC group were decreased, while both OCN and RhoA expression were significantly increased and the Ephrin-B2 downstream factor Grb4 in in GC group was increased after icariin treatment. Conclusion. Icariin could improve the characteristics of OP through regulating the balance of the EphB4/Ephrin-B2 pathway. Further preclinical trial is needed to provide certainty of clinical benefits for OP patients.
\end{abstract}

\section{Introduction}

Osteoporosis (OP) is a skeletal disorder characterized by decreased bone strength and increased risk of fracture. OP can be divided into primary and secondary damage, and the secondary damage is mainly caused by any disease or drug that affects bone formation, among which glucocorticoid (GC) is the most important risk factor for OP. GC is widely used in the clinic due to its anti-inflammatory and immunosuppressive effects. However, long-term use of GC usually resulted in a 30\% 50\% incidence of osteoporotic fractures, which seriously affects the quality of life of patients [1-5].

A number of signal pathways have been found participating in osteoblasts and osteoclasts in bone formation, and
EphB4/Ephrin-B2 is the bidirectional signal axis that is separately expressed in the osteoblast and osteoclast cells [6], which produce positive or reverse signals. Ephrin-B2 inhibits osteoclast function through c-Fos/NFATc1, while EphB4 factor promotes osteoblast function by osteogenesis Dlx5, Osx, and Runx2 [7]. Similarly, Sema3A/Nrp, another signaling pathway between osteoblasts and osteoclasts, has been found to inhibit RANKL-induced osteoclast differentiation by inhibiting ITAM and RhoA signaling pathways [8]. Also, KEGG analysis found that EphB4 could participate in cytoskeleton development through the RhoA signaling pathway, while Ephrin-B2 could bind to Grb4 to generate inhibitory regulation. Nevertheless, the destruction of such signal axis causes functional changes of osteoblasts and 
osteoclasts, which may further lead to the formation barriers of osteocytes and the reduction of mineral deposits and increase the risk of formation of OP and fracture.

Recent studies have suggested that icariin could protect GC-induced OP [9], and icariin is found to participate in bone metabolism and synthesis through MAPK, RUN2, Rankl, and Wnt-catenin pathways and improve the differentiation of osteoblast precursor cells and osteoblast progression [10-15]. Interestingly, EphB4/Ephrin-B2 and Notch signaling pathways were revealed to play an important role in SRY-mediated male-specific artery formation during testosterone-induced XY gonad development [16]. However, icariin is a testosterone analogue [17], so it remains unclear whether the EphB4/Ephrin-B2 signaling axis is the key signaling mechanism for icariin. In the present study, we investigated the potential mechanism of icariin in GC-induced OP.

\section{Materials and Methods}

2.1. Animals. The C57/BL6 male mice (8 weeks, $21 \pm 1.8 \mathrm{~g}$ ) were used, supplied by the Experimental Animal Center of Tongji Medical College of Huazhong University of Science and Technology. The experiment complied the Regulations for the Administration of Affairs Concerning Experimental Animals by China (revised 2017). The study was approved by the Local Animal Ethics Committee of Wuhan First Hospital. The mice were randomly divided into 4 groups with 6 mice. Each group was treated for 60 days and followed by gavage for following four weeks according to the same protocol in [18]. The control group received continuous subcutaneous injection of normal saline at $5 \mathrm{mg} / \mathrm{kg}$, followed by intragastric gavage with normal saline at $250 \mathrm{mg} / \mathrm{kg} /$ day; in the experimental group, $5 \mathrm{mg} / \mathrm{kg}$ prednisone sustained release agent was continuously injected subcutaneously, and the same amount of normal saline was gavage; in the drug treatment group, $5 \mathrm{mg} / \mathrm{kg}$ prednisone sustained release was injected subcutaneously continuously, and $250 \mathrm{mg} / \mathrm{kg} / \mathrm{day}$ icariin (PS13012801, Purity: 99.50\%, Chengdu PUSH BioTechnology Co., Ltd.) was gavage. The positive control group was subcutaneously injected with the same amount of normal saline and gavage of icariin.

2.2. Histology Analysis. The right femurs and tibias were obtained and fixed at $4 \%$ formalin for at least $24 \mathrm{hrs}$ and then immersed in 10\% EDTA for 4 weeks, followed by embedding in paraffin. The tissues were cut to $4-\mu \mathrm{m}$ on slides. Following $\mathrm{H} \& \mathrm{E}$ staining, the sections were observed with an inverted microscope (Olympus, Tokyo, Japan).

2.3. Immunohistochemistry (IHC) Staining. The sections were deparaffinized in xylene followed by hydration in ethanol. Antigen retrieval was performed using citrate $(\mathrm{pH}$ 6.0 ) in a microwave. Then, the samples were blocked in bovine serum albumin for 1 hour. Primary antibodies EphB4 (bs-10659R) and Ephrin-B2 (bs-6046R), purchased from Beijing Bioss Biological Technology (Beijing, China), were incubated $\left(1: 200,4^{\circ} \mathrm{C}\right.$, overnight), followed by incubation with a secondary antibody (HRP, DAKO, Carpinteria, CA, USA) for 1 hour. Diaminobenzidine (DAB-DAKO, Carpinteria, CA, USA) was used for positive visualization. The sections were observed with an inverted microscope (Olympus, Tokyo, Japan).

2.4. Western Blot (WB) Analysis. Whole proteins were isolated according to the kit protocol (KeyGen Biotech, Nanjing, China), and protein was then separated by $10 \%$ SDS-PAGE gels and transferred to polyvinyl difluoride (PVDF) membranes. Nonfat milk diluted in TBST was used for blocking for 1 hour at room temperature. Primary antibodies Grb4 (Proteintech, 10206-1-AP), RhoA (Proteintech, 10749-1-AP), and OCN (Proteintech, 23418-1-AP) (all from Wuhan SanYing, Wuhan) were incubated overnight at $4^{\circ} \mathrm{C}$ and then incubated with the anti-rabbit antibody for 2 hours at room temperature. The proteins were detected by the ECL detection kit, and relative expression levels were normalized to $\beta$-actin.

2.5. Micro-CT Imaging. After the mice in each group were sacrificed, the right tibia was quickly removed, and then the attached soft tissue was removed and the bone tissue was fixed in a $4 \%$ paraformaldehyde solution. The specimen was placed in a sample cup and fixed, and the trabecular microstructure of the tibia was scanned using an in vitro microCT tomography technique (SkyScan 1176, Institute of Hydrobiology, Chinese Academy of Sciences). The scanning conditions were set to a voltage of $70 \mathrm{kV}$, a scanning current of $200 \mu \mathrm{A}$, a layer spacing of $14.80 \mu \mathrm{m}$, a planar resolution of $300 \mathrm{~ms}$, and a continuous scanning of about 808 layers. After the scan is completed, the sacral enlargement and the backbone area are selected as the region of interest (ROI). After the three-dimensional reconstruction threshold is set, the three-dimensional reconstruction of the ROI is performed. The airborne software CTan analysis is used. The main detection parameters are as follows: (1) bone mineral density parameters: BMD (bone mineral density), TMC (tissue mineral content), and BMC (bone mineral content); (2) bone structure parameters: BV/TV (bone volume/total volume), Tb.N (trabecular bone number), and Tb.Th (trabecular thickness) [19].

2.6. Statistical Analyses. One-way ANOVA followed by Tukey post hoc test was used for statistical analyses. The data were expressed as means $\pm \mathrm{SD}$. $P$ values $<0.05$ were considered as statistically significant.

\section{Results}

3.1. Icariin Alleviates GC-Induced OP. HE staining (Figure 1) showed abundant, continuous, and dense trabecular bone in normal mice, but the GC treatment group showed a larger marrow cavity and a decreasing number of trabecular bones, revealing the typical characteristics of osteoporosis. The icariin treatment revealed reverses of those osteoporosis 


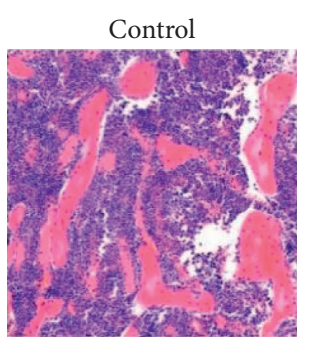

(a)

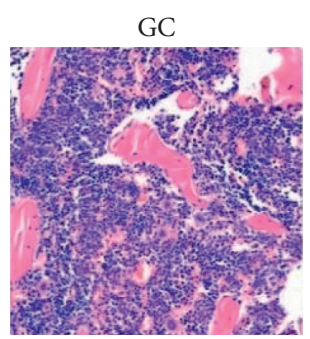

(b)

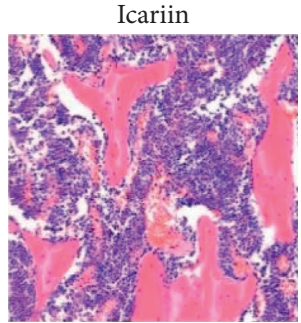

(c)

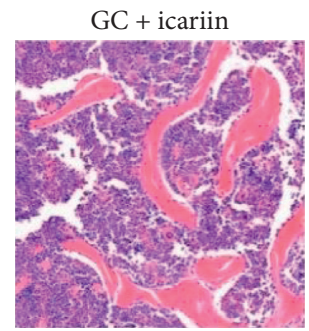

(d)

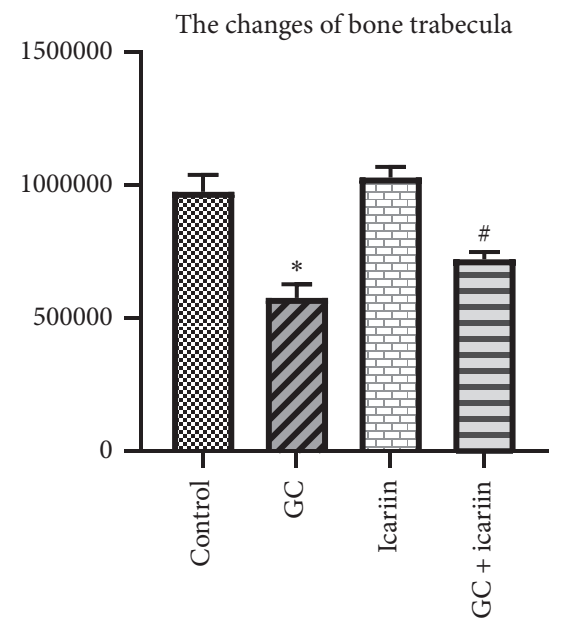

(e)

Figure 1: Icariin can alleviate the GC-induced OP. HE staining (200x) of control group (a), GC group (b), icariin group (c), and icariin treatment of GC-induced OP group (d). The GC treatment group showed a larger marrow and a decreasing number of trabecular bone; icariin treatment can reverse those changes (e). ${ }^{*} P<0.05$ vs the control group and ${ }^{\#} P<0.05$ vs the GC group.

changes, suggesting icariin along having no changes as compared with the normal mice.

\subsection{Icariin Improves Trabecular Bone Parameters in GC-In-} duced OP. Trabecular bone parameters were analyzed by using micro-CT (Figure 2), and the results showed that GC treatment had significantly negative effects on BV/TV, Tb.N, Tb.Th, BMD, TMC, and BMC, while icariin treatment significantly reduced the negative effects of structural properties of the trabecular bone on BV/TV, Tb.N, BMD, TMC, and BMC. Icariin along had no structural changes as compared with the normal mice.

\subsection{Icariin Elevates EphB4 While Suppressing Ephrin-B2} Expression in GC-Induced OP. To further explore the relationship between EphB4 and Ephrin-B2 in GC-induced OP, IHC staining (Figure 3) was used, revealing GC-induced Ephrin-B2 expression, while after icariin treatment, EphrinB2 expression was significantly decreased. We further found that GC can suppress EphB4 expression, while after icariin treatment, Ephrin-B2 expression was significantly increased. The WB results also confirmed the protein expression trend.

3.4. Icariin Alleviates GC-Induced OP through the EphB4/ Ephrin-B2 Axis. We also examined the signaling pathway involved in GC-induced OP. The WB results (Figure 4) showed that the osteoblast marker OCN and the EphB4 downstream factor RhoA in the GC group were decreased, while after icariin treatment, both OCN and RhoA expression were significantly increased. Furthermore, we found that the Ephrin-B2 downstream factor Grb4 in the GC group was decreased, while after icariin treatment, Grb4 expression was significantly increased. The results suggested that icariin might regulate the balance of the EphB4/EphrinB2 axis, thus promoting the recovery of GC-induced OP.

\section{Discussion}

The clinical treatment for GC-induced OP relies on drugs including calcium supplementation, vitamin D, bisphosphonates, hormones, calcitonin, and fluoride. However, the effect of drug therapy is still unsatisfactory [20]. Icariin, a natural product from traditional Chinese medicine (TCM), has been studied for treatment of osteoporosis [21], and its pharmacological function remains unclear. In the present study, we found that icariin could alleviate GC-induced OP through regulating the balance of the EphB4/Ephrin-B2 axis, thus enhancing the recovery of OP.

GC is an important pathogenetic factor for OP and affects the differentiation and longevity of osteoclasts and osteoblasts through redox pathways [22]. GC reduces replication of osteoblastic lineage cells and inhibits osteogenic differentiation or mineralization, resulting in the osteoblasts 


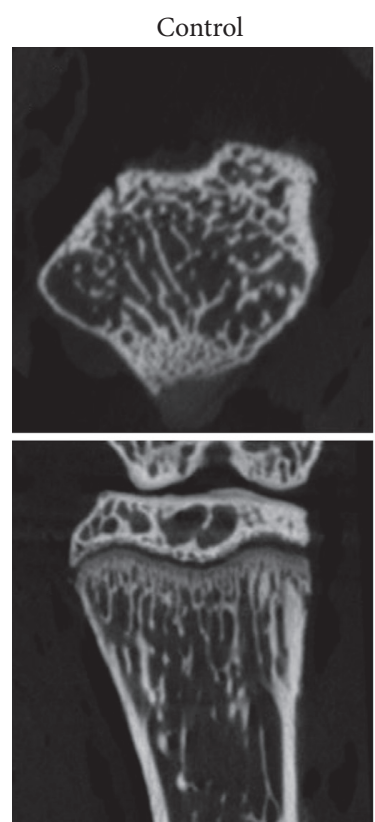

(a)
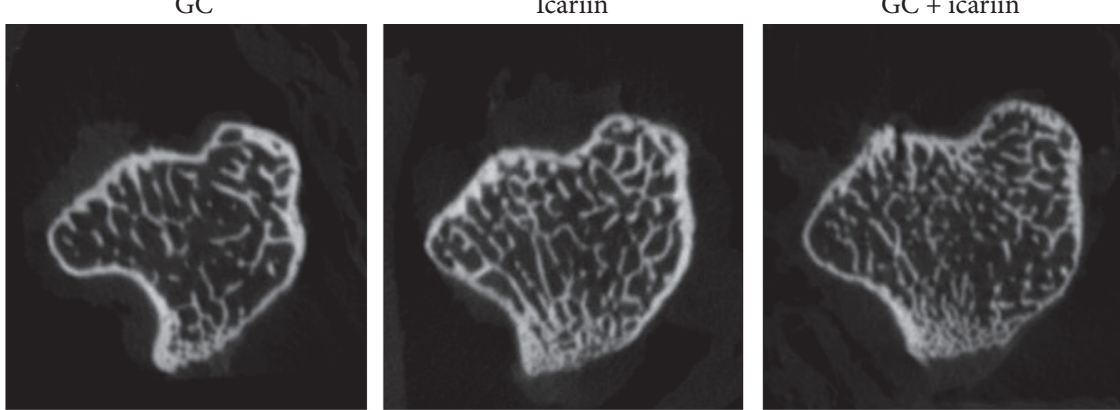

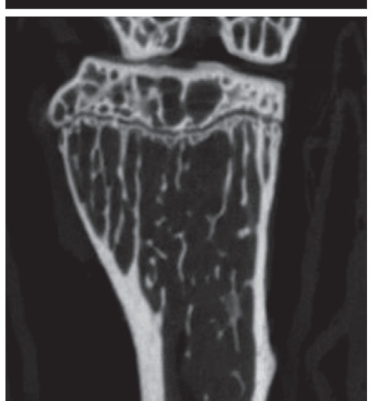

(b)

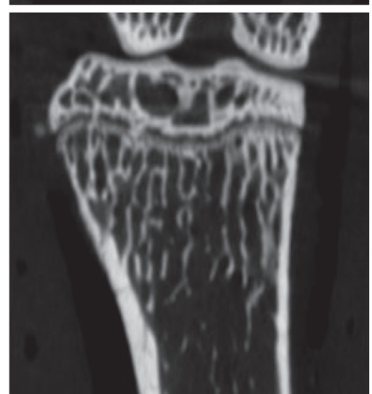

(c)

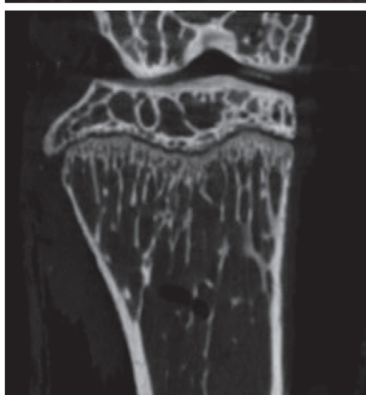

(d)

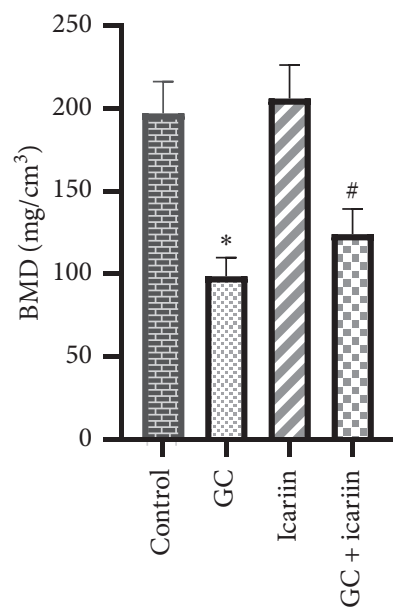

(e)

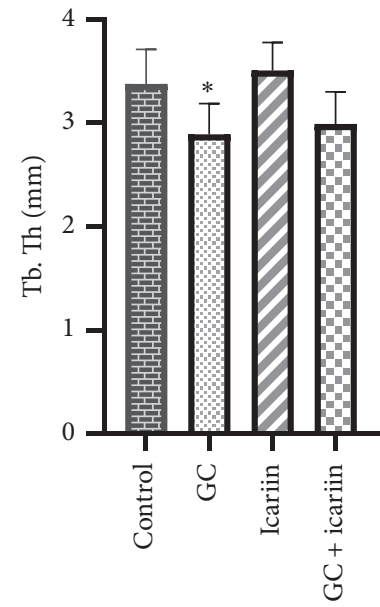

(h)

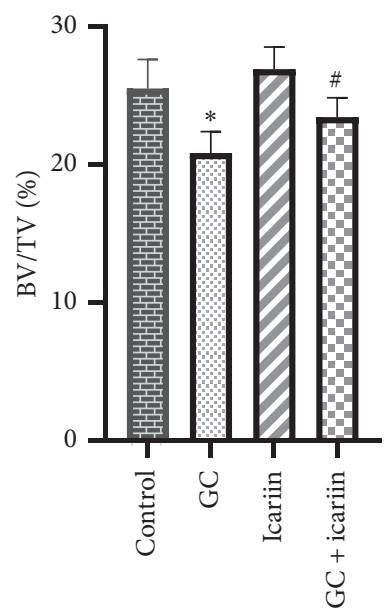

(f)

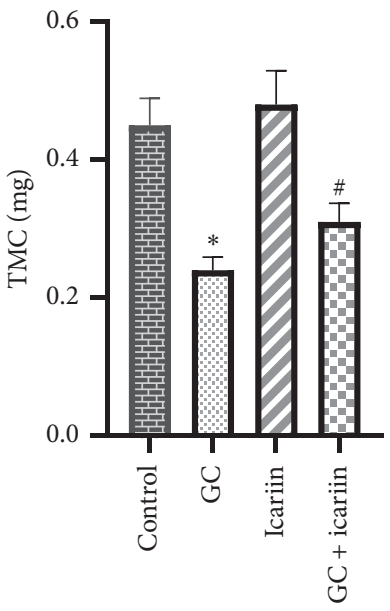

(i)

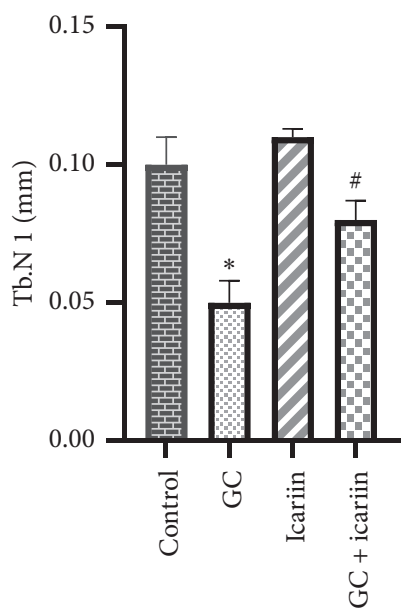

(g)

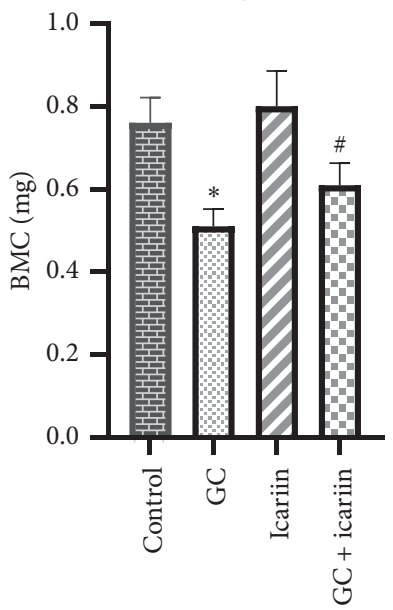

(j)

FIGURE 2: Icariin can improve trabecular bone parameters in GC-induced OP. Micro-CT was used to test trabecular bone parameters in the control group (a), GC group (b), icariin group (c), and icariin treatment of GC-induced OP group (d). Results on bone mineral density $(\mathrm{BMD})(\mathrm{e})$, bone volume fraction (BV/TV) $(\mathrm{f})$, trabecular number (Tb.N) (g), trabecular thickness (Tb.Th) (h), tissue mineral content (TMC) (i), and trabecular bone mineral content (Tb.BMC) (j). The GC treatment made significant negative effects on microstructural quantitative parameters, while icariin can prevent those changes. ${ }^{*} P<0.05$ vs the control group and ${ }^{\#} P<0.05$ vs the GC group. 

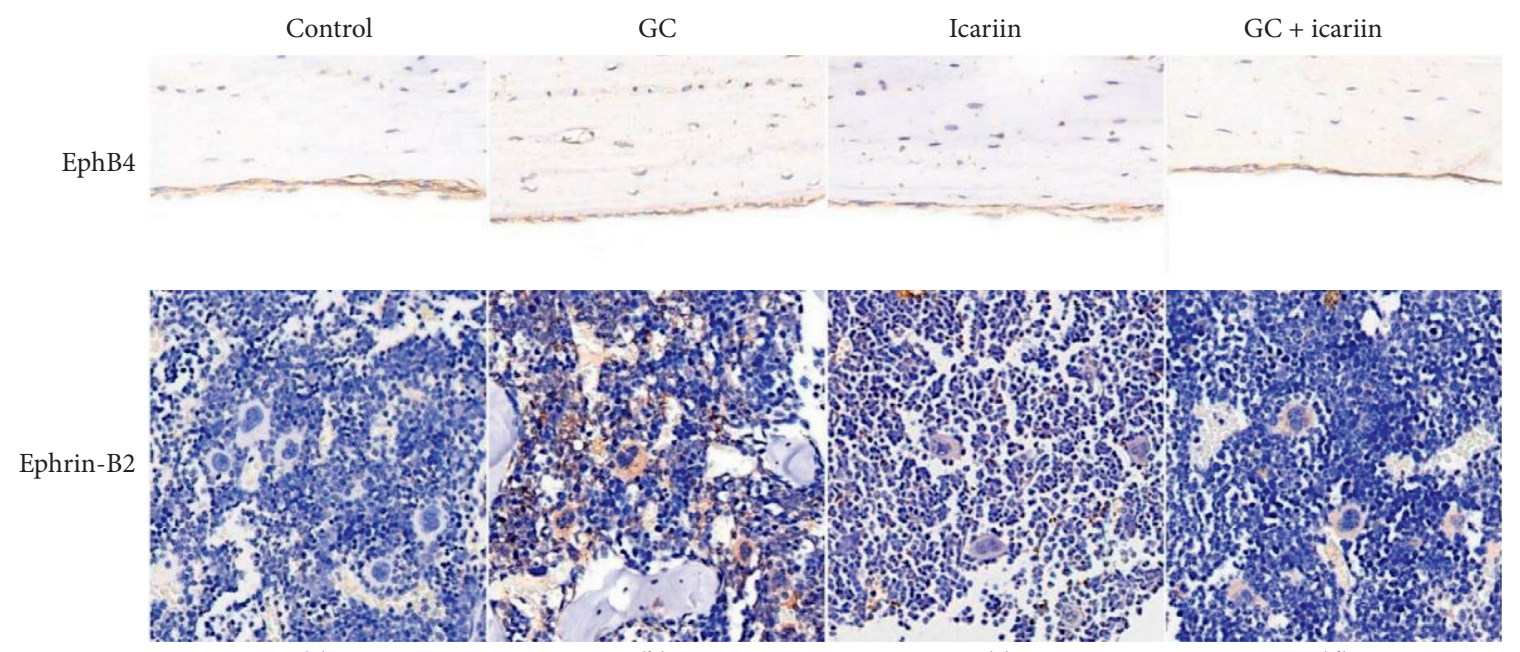

(a)

(b)

(c)

(d)

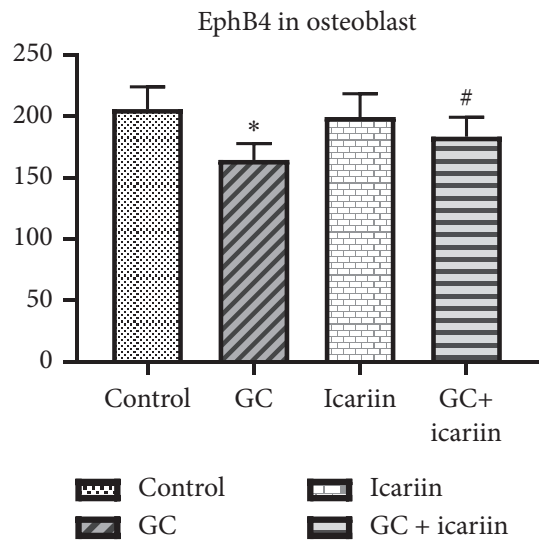

(e)

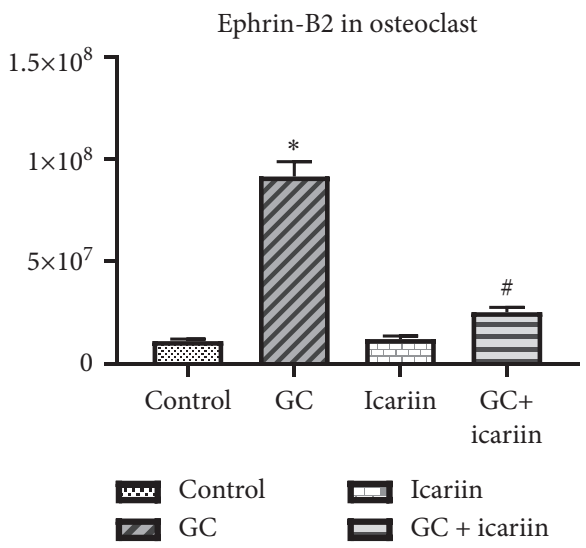

(f)

FIGURE 3: Icariin can promote EphB4 while suppress Ephrin-B2 expression in GC-induced OP. IHC staining of Ephrin-B2 and EphB4 in the control group (a), GC group (b), icariin group (c), and icariin treatment of GC-induced OP group (d). The WB results showed that the GC treatment decreased EphB4 expression and increased Ephrin-B2 expression compared with the control group, and the GC + icariin group showed the opposite result compared with the GC group (e and f). ${ }^{*} P<0.05$ vs the control group and ${ }^{\#} P<0.05$ vs the GC group.

in an immature state, thereby reducing their numbers and function. GC can also indirectly inhibit osteogenic differentiation and promote adipogenic differentiation in stromal cells [23]. Moreover, GC also increases the function and number of osteoclasts and their survival time, such as caspase- 3 can prolong its life and M-CSF can enhance its activity [24, 25].

The Eph receptor is a primary component of the tyrosine kinase receptor family. EphB4/Ephrin-B2 signaling is an important axis which regulates the functional balance of osteoblasts and osteoclasts [26]. The regulation was via the transmembrane ligand, such as Ephrin-2 on osteoclasts and receptor tyrosine kinase and EphB4 on osteoblasts [7]. Previous studies suggested that EphB4 receptor activation by Ephrin-B2 could affect resorption factors and their activities in the subchondral bone [27]. Ephrin-B2 treatment of osteoarthritic chondrocytes increases the gene expression levels of type II collagen in these cells [28], suggesting that Ephrin-B2 also plays a role in regulating chondrocyte differentiation. It was also reported that osteoclastogenesis could be attenuated through bidirectional EphB4/Ephrin-B2 signaling in vitro [29], while another study found that Claudin 11 may regulate bone homeostasis via bidirectional EphB4/Ephrin-B2 signaling [30]. In the present study, we found that GC treatment could induce typical characteristics of osteoporosis, while icariin treatment reversed those osteoporosis changes and demonstrated a therapeutic effect of icariin on GC-induced OP. Further study found that both EphB4 and Ephrin-B2 participated in the pathological changes of OP, which is consistent with previous studies that EphB4/Ephrin-B2 signaling also promotes the differentiation of osteoblasts and osteoclasts [26], indicating an imbalance expression of EphB4 and Ephrin-B2 could be a potential mechanism which might disturb the function of osteoblasts and osteoclasts.

Moreover, through KEGG analysis, we found that EphB4 could act on the RhoA signaling pathway; i.e., Ephrin-B2 could bind to Grb4 and might further regulate cytoskeleton development. To test whether RhoA and Grb4 pathways are involved in the EphB4/Ephrin-B2 signaling, we detected 

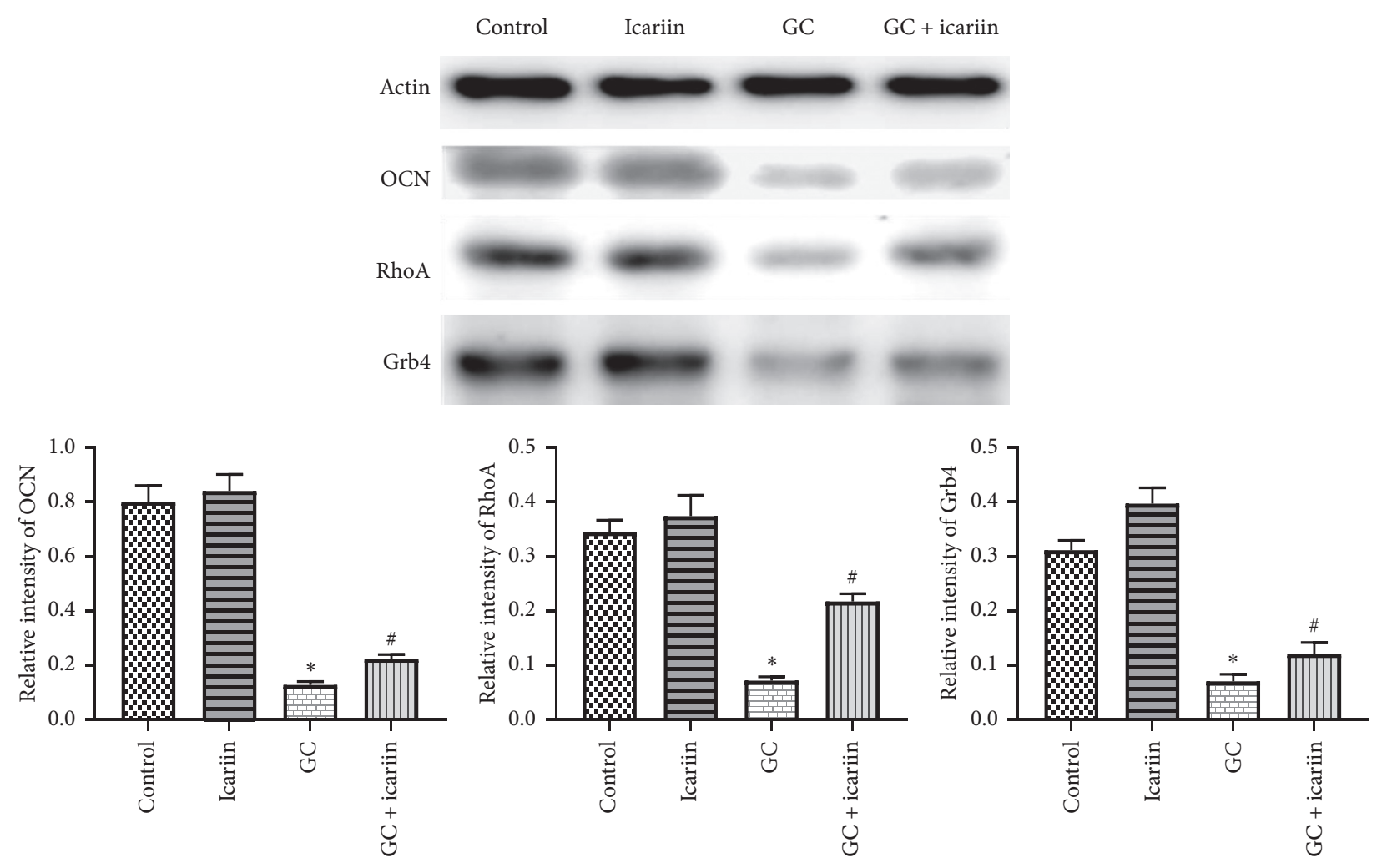

Control GC Icariin GC+icariin

Actin

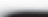

Actin
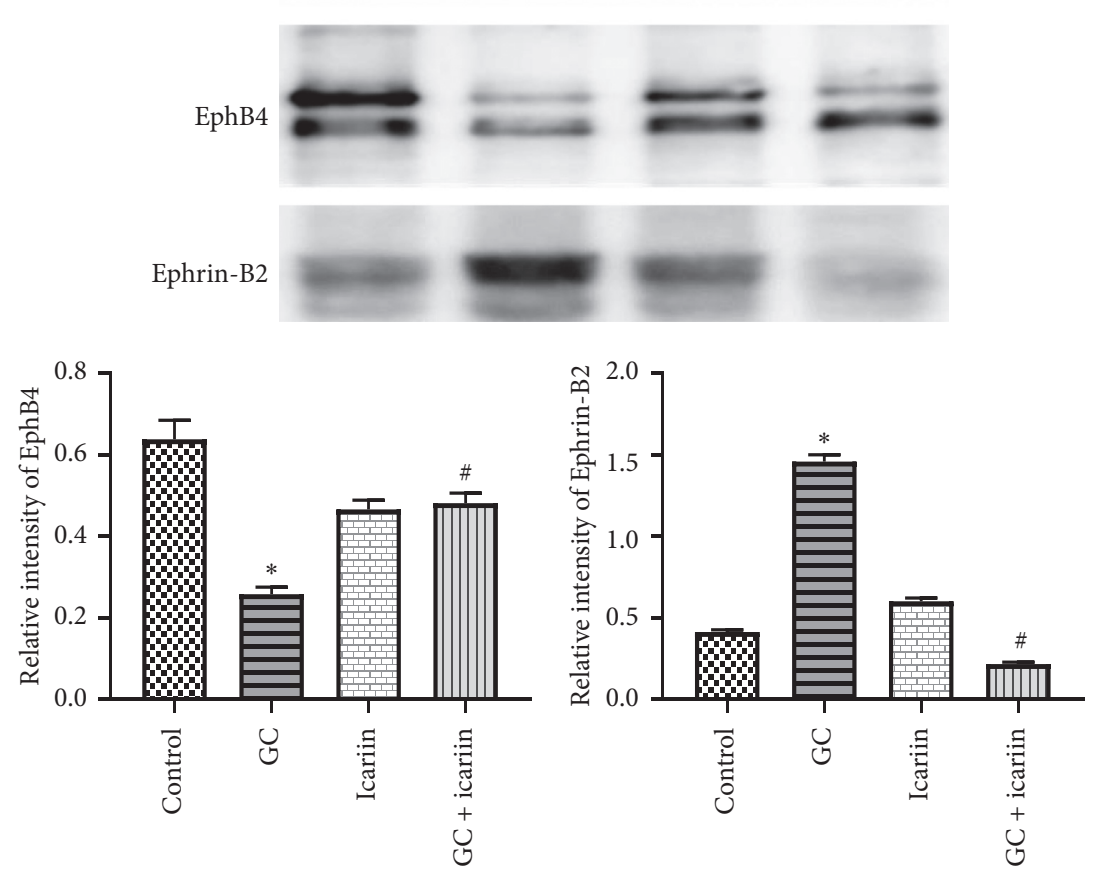

FIgURE 4: Icariin alleviates GC-induced OP through the EphB4/Ephrin-B2 axis. WB analysis of the osteoblast marker OCN, EphB4 downstream factor RhoA, and Ephrin-B2 downstream factor Grb4. The result showed that the osteoblast marker OCN, the EphB4 downstream factor RhoA, and the Ephrin-B2 downstream factor Grb4 in the GC group were decreased, while after icariin treatment, their expressions were all significantly increased. Protein expression levels were normalized to $\beta$-actin. ${ }^{*} P<0.05$ vs the control group and ${ }^{\#} P<0.05$ vs the GC group. 
their protein levels and found lower expression of RhoA but higher Grb4 levels in GC-induced OP, while treatment with icariin reversed the expression, which implied that EphB4 could further inhibit osteoblasts through the RhoA signaling pathway, while Ephrin-B2 regulated Grb4 to promote osteoclast function. As their functional properties are similar to testosterone [17], our findings provided new evidence that icariin regulates the expression of EphB4/Ephrin-B2 and maintains the functional balance of osteoblasts and osteoclasts through RhoA and Grb4, respectively, to protect the bone structure.

\section{Conclusions}

In the present study, we found icariin could improve characteristics of OP through regulating the balance of EphB4/Ephrin-B2 to protect the bone structure. A further preclinical trail might be needed to provide certainty of clinical benefits for OP patients.

$\begin{array}{ll}\text { Abbreviations } \\ \text { OP: } & \text { Osteoporosis } \\ \text { GC: } & \text { Glucocorticoid } \\ \text { IHC: } & \text { Immunohistochemistry } \\ \text { WB: } & \text { Western blot } \\ \text { ROI: } & \text { Region of interest } \\ \text { BMD: } & \text { Bone mineral density } \\ \text { TMC: } & \text { Tissue mineral content } \\ \text { BMC: } & \text { Bone mineral content } \\ \text { BV/TV: } & \text { Bone volume/total volume } \\ \text { Tb.N: } & \text { Trabecular bone number } \\ \text { Tb.Th: } & \text { Trabecular thickness. }\end{array}$

\section{Data Availability}

The data used to support the findings of this study are included within the article. Any further data can be made available from the corresponding author upon request.

\section{Conflicts of Interest}

The authors declare that they have no conflicts of interest.

\section{Authors' Contributions}

M.H contributed to the design of the work, data collection, analysis and interpretation of the data, statistical analysis, and drafting the manuscript. Y.W contributed to the critical revision of the manuscript and provided financial support. R.P contributed to project design and critical revision of the manuscript. All authors read and approved the final manuscript.

\section{Acknowledgments}

This project was supported by the Key Area R\&D Project of Guangdong Province (2020B020221001), Guangdong Provincial Key Laboratory of Applied Botany, South China Botanical Garden, Chinese Academy of Sciences
(AB2018027), and the Science and Technology Department of Hubei Province, Natural Science Foundation of Hubei Province (2018CFB695).

\section{References}

[1] L. Buckley and M. B. Humphrey, "Glucocorticoid-induced osteoporosis," New England Journal of Medicine, vol. 379, no. 26, pp. 2547-2556, 2018.

[2] A. Klibanski, L. Adams-Campbell, T. L. Bassford et al., "Osteoporosis prevention, diagnosis, and therapy," Journal of the American Medical Association, vol. 285, no. 6, pp. 785795, 2001.

[3] R. S. Weinstein, "Glucocorticoid-induced osteoporosis and osteonecrosis," Endocrinology and Metabolism Clinics of North America, vol. 41, no. 3, pp. 595-611, 2012.

[4] E. G. Matzkin, M. DeMaio, J. F. Charles, and C. C. Franklin, "Diagnosis and treatment of osteoporosis," Journal of the American Academy of Orthopaedic Surgeons, vol. 27, no. 20, pp. e902-e912, 2019.

[5] J. Compston, "Glucocorticoid-induced osteoporosis: an update," Endocrine, vol. 61, no. 1, pp. 7-16, 2018.

[6] Y. Tazaki, K. Sugitani, K. Ogai et al., "RANKL, Ephrin-Eph and Wnt10b are key intercellular communication molecules regulating bone remodeling in autologous transplanted goldfish scales," Comparative Biochemistry and Physiology Part A: Molecular \& Integrative Physiology, vol. 225, pp. 4658, 2018.

[7] X. Chen, Z. Wang, N. Duan, G. Zhu, E. M. Schwarz, and C. Xie, "Osteoblast-osteoclast interactions," Connective Tissue Research, vol. 59, no. 2, pp. 99-107, 2018.

[8] M. Hayashi, T. Nakashima, M. Taniguchi, T. Kodama, A. Kumanogoh, and H. Takayanagi, "Osteoprotection by semaphorin 3A," Nature, vol. 485, no. 7396, pp. 69-74, 2012.

[9] R. Feng, L. Feng, Z. Yuan et al., "Icariin protects against glucocorticoid-induced osteoporosis in vitro and prevents glucocorticoid-induced osteocyte apoptosis in vivo," Cell Biochemistry and Biophysics, vol. 67, no. 1, pp. 189-197, 2013.

[10] Y. Liu, L. Huang, B. Hao et al., "Use of an osteoblast overload damage model to probe the effect of icariin on the proliferation, differentiation and mineralization of MC3T3-E1 cells through the Wnt/ $\beta$-catenin signalling pathway," Cellular Physiology and Biochemistry, vol. 41, no. 4, pp. 1605-1615, 2017.

[11] Y. Wu, L. Xia, Y. Zhou, Y. Xu, and X. Jiang, "Icariin induces osteogenic differentiation of bone mesenchymal stem cells in a MAPK-dependent manner," Cell Proliferation, vol. 48, no. 3, pp. 375-384, 2015.

[12] Z. Wu, L. Ou, C. Wang et al., "Icaritin induces MC3T3-E1 subclone14 cell differentiation through estrogen receptormediated ERK1/2 and p38 signaling activation," Biomedicine \& Pharmacotherapy, vol. 94, pp. 1-9, 2017.

[13] S. Zhang, P. Feng, G. Mo et al., "Icariin influences adipogenic differentiation of stem cells affected by osteoblast-osteoclast co-culture and clinical research adipogenic," Biomedicine \& Pharmacotherapy, vol. 88, pp. 436-442, 2017.

[14] C. V. Pham, T. T. Pham, T. T. Lai et al., "Icariin reduces bone loss in a Rankl-induced transgenic medaka (Oryzias latipes) model for osteoporosis," Journal of Fish Biology, 2020, In press.

[15] X. Huang, X. Wang, Y. Zhang et al., "Absorption and utilisation of epimedin $\mathrm{C}$ and icariin from Epimedii herba, and the regulatory mechanism via the $\mathrm{BMP} 2 /$ Runx2 signalling 
pathway," Biomedicine \& Pharmacotherapy, vol. 118, Article ID 109345, 2019.

[16] J. Brennan, J. Karl, and B. Capel, "Divergent vascular mechanisms downstream of Sry establish the arterial system in the XY gonad," Developmental Biology, vol. 244, no. 2, pp. 418-428, 2002.

[17] Z.-B. Zhang and Q.-T. Yang, "The testosterone mimetic properties of icariin," Asian Journal of Andrology, vol. 8, no. 5, pp. 601-605, 2006.

[18] G. Chen, C. Wang, J. Wang et al., "Antiosteoporotic effect of icariin in ovariectomized rats is mediated via the Wnt/ $\beta$-catenin pathway," Experimental and Therapeutic Medicine, vol. 12, no. 1, pp. 279-287, 2016.

[19] Y. Ma, H. Yang, and J. Huang, "Icariin ameliorates dexamethasoneinduced bone deterioration in an experimental mouse model via activation of microRNA186 inhibition of cathepsin K," Molecular Medicine Reports, vol. 17, no. 1, pp. 1633-1641, 2018.

[20] U. Massafra, S. Migliaccio, C. Bancheri et al., "Approach in glucocorticoid-induced osteoporosis prevention: results from the Italian multicenter observational EGEO study," Journal of Endocrinological Investigation, vol. 36, no. 2, pp. 92-96, 2013.

[21] J. An, H. Yang, Q. Zhang et al., "Natural products for treatment of osteoporosis: the effects and mechanisms on promoting osteoblast-mediated bone formation," Life Sciences, vol. 147, pp. 46-58, 2016.

[22] S. Zhang, D. Li, J.-Y. Yang, and T.-B. Yan, "Plumbagin protects against glucocorticoid-induced osteoporosis through Nrf-2 pathway," Cell Stress and Chaperones, vol. 20, no. 4, pp. 621-629, 2015.

[23] L. Han, B. Wang, R. Wang, S. Gong, G. Chen, and W. Xu, "The shift in the balance between osteoblastogenesis and adipogenesis of mesenchymal stem cells mediated by glucocorticoid receptor," Stem Cell Research \& Therapy, vol. 10, no. 1, p. 377, 2019.

[24] H.-J. Kim, "New understanding of glucocorticoid action in bone cells," BMB Reports, vol. 43, no. 8, pp. 524-529, 2010.

[25] L. I. Plotkin, N. Bivi, and T. Bellido, "A bisphosphonate that does not affect osteoclasts prevents osteoblast and osteocyte apoptosis and the loss of bone strength induced by glucocorticoids in mice," Bone, vol. 49, no. 1, pp. 122-127, 2011.

[26] Y. Wang, A. Menendez, C. Fong, H. Z. ElAlieh, W. Chang, and D. D. Bikle, "EphrinB2/EphB4 mediates the actions of IGF-I signaling in regulating endochondral bone formation," Journal of Bone and Mineral Research, vol. 29, no. 8, pp. 1900-1913, 2014.

[27] S. Kwan Tat, J.-P. Pelletier, N. Amiable et al., "Activation of the receptor EphB4 by its specific ligand ephrin B2 in human osteoarthritic subchondral bone osteoblasts," Arthritis \& Rheumatism, vol. 58, no. 12, pp. 3820-3830, 2008.

[28] S. Kwan Tat, J.-P. Pelletier, N. Amiable, C. Boileau, M. Lavigne, and J. Martel-Pelletier, "Treatment with ephrin B2 positively impacts the abnormal metabolism of human osteoarthritic chondrocytes," Arthritis Research \& Therapy, vol. 11, no. 4, p. R119, 2009.

[29] Y. W. Ge, Z. Q. Liu, Z. Y. Sun et al., "Titanium particlemediated osteoclastogenesis may be attenuated via bidirectional ephrinb2/ephB4 signaling in vitro," International Journal of Molecular Medicine, vol. 42, no. 4, pp. 2031-2041, 2018.

[30] J. M. Baek, Y.-H. Cheon, S. C. Kwak et al., "Claudin 11 regulates bone homeostasis via bidirectional EphB4-EphrinB2 signaling," Experimental \& Molecular Medicine, vol. 50, no. 4, pp. 1-18, 2018. 